\title{
Classification and Identification of Xanthomonas translucens Isolates, Including Those Pathogenic to Ornamental Asparagus
}

\author{
J. L. W. Rademaker, D. J. Norman, R. L. Forster, F. J. Louws, M. H. Schultz, and F. J. de Bruijn
}

First, fifth, and sixth authors: MSU-DOE Plant Research Laboratory, Michigan State University, East Lansing 48824; second author: MidFlorida Research and Education Center, University of Florida, Apopka 32703; third author: University of Idaho Research and Extension Center, Kimberly 83341; fourth author: Department Plant Pathology, North Carolina State University, Raleigh 27695; and sixth author: Department of Microbiology and NSF Center for Microbial Ecology, Michigan State University.

Current address of J. L. W. Rademaker: NIZO food research, Health \& Safety Division, P.O. Box 20, 6710 BA Ede, The Netherlands.

Current address of F. J. de Bruijn: INRA-CNRS Laboratoire de Biologie Moleculaire de Relations Plantes-Microorganismes Chemin de Borde-Rouge, Auzeville B.P. 27, 31326 Castanet-Tolosan Cedex, France.

Accepted for publication 29 March 2006.

\section{ABSTRACT}

Rademaker, J. L. W., Norman, D. J., Forster, R. L., Louws, F. J., Schultz, M. H., and de Bruijn, F. J. 2006. Classification and identification of Xanthomonas translucens isolates, including those pathogenic to ornamental asparagus. Phytopathology 96:876-884.

In order to confirm and refine the current classification scheme of Xanthomonas translucens and to identify novel strains from ornamental asparagus, a collection of field and reference strains was analyzed. Reppolymerase chain reaction (PCR) genomic fingerprint profiles were generated from 33 isolates pathogenic to asparagus as well as $61 \mathrm{X}$. translucens reference strains pathogenic to cereals and grasses. Amplified ribosomal gene restriction analysis profiles were obtained from most of these and 29 additional Xanthomonas reference strains. Rep-PCR genomic fingerprint profiles of all strains were compared with those in a large Xanthomonas database using computer-assisted analysis. Rep-PCR genomic fingerprinting facilitated the characterization and discrimination of $X$. translucens, including the pathovars arrhenatheri, graminis, phlei, phleipratensis, and poae, as well as a number of strains received as

Identification and classification of Xanthomonas translucens variants (pathovars) pathogenic on members of the families Gramineae and Poaceae (cereals and grasses) is confusing. The pathovar classification relies on the host range of the pathogen, and is poorly defined due to variation in host-pathogen interactions (8). Moreover, pathogenicity tests are cumbersome, laborious, time consuming, costly, and have limited reproducibility. Isolates with an unclear taxonomic position that display a distinct pathogenicity or obtained from novel hosts are often difficult to classify at the subspecific (pathovar) level due to limitations in examining a comprehensive host range. A complicating factor is that host specificity coincides with different genomic backgrounds ([geno]species, polymerase chain reaction [PCR] fingerprint types, or both), such as in xanthomonads associated with pepper and tomato lesions $(2,26,29,35)$, rice $(6,26)$, and ornamentals $(35)$ that were differentiated with the advent of PCR-based genomic typing methods (15).

In serological tests using polyclonal and monoclonal antibodies, the $X$. translucens pathovars cerealis, hordei, secalis, translucens, and undulosa form a homogeneous group (5), and

Corresponding author: J. L. W. Rademaker

E-mail address: Rademake@dds.nl or Jan.Rademaker@nizo.nl

DOI: 10.1094/PHYTO-96-0876

C 2006 The American Phytopathological Society
$X$. translucens pv. cerealis. Strains received as pathovars hordei, secalis, translucens, undulosa, and other cerealis strains were grouped in two subclusters that correspond to the recently redefined pathovars $X$. translucens pvs. undulosa and translucens. All 33 novel isolates from ornamental asparagus (tree fern; Asparagus virgatus) were identified as $X$. translucens pv. undulosa. Moreover, a unique amplified small subunit ribosomal gene $M s p \mathrm{I} / A l u \mathrm{I}$ restriction profile specific for all $X$. translucens strains tested, including those pathogenic to asparagus, allowed discrimination from all other Xanthomonas spp. Although phage tests were inconclusive, the classification of the asparagus strains within the $X$. translucens complex was supported by pathogenicity assays in which all the isolates from ornamental asparagus induced watersoaking on wheat. Surprisingly, several $X$. translucens reference strains affected asparagus tree fern as well. That the novel asparagus isolates belong to $X$. translucens pv. undulosa is extraordinary because all hosts of $X$. translucens pathovars described to date belong only to the families Gramineae and Poaceae, whereas asparagus belongs to the phylogenetically distant family Liliaceae. cannot be differentiated by FAME analysis (31). The polyphasic approach of Vauterin et al. (38), combining sodium dodecyl sulfate polyacrylamide gel electrophoresis and FAME analysis with DNA-DNA hybridization experiments, has provided a more robust and genotypic framework. Computer-assisted analysis of protein profiles has permitted the differentiation of pathovars arrhenatheri, graminis, phlei, phleipratensis, and poae (38), but not the other $X$. translucens pathovars.

Extensive comparison of small-subunit ribosomal DNA (SSU rDNA) sequences has revealed limited variability of this sequence within the genus $(12,19)$. Therefore, SSU rDNA sequences have limited value in differentiating Xanthomonas strains even at the species level. Moreover, the phylogenetic structure of the genus is only loosely related to phytopathogenic specialization $(35,36)$. However, Bragard et al. (3) did report a correlation between pathogenic and genotypic analyses (i.e., amplified fragment length polymorphism [AFLP] and ribotype analyses) in a comprehensive study of $X$. translucens using a collection of 68 strains, including several pathovar reference strains. Additionally, a high correlation between AFLP and rep-PCR genomic fingerprinting and DNADNA homology studies was described (26), and a large database of rep-PCR genomic fingerprint profiles for classification and identification of xanthomonads was generated (29).

Recently, the dark watersoaked lesions on stems of ornamental asparagus tree fern (Asparagus virgatus) from commercial nurseries in two counties in Florida was reported for the first time 
(22). The 33 bacterial isolates obtained from the lesions were identified as xanthomonads using conventional biochemical tests, Biolog, and FAME analysis (22).

In the present study, in order to identify novel strains from ornamental asparagus and refine the current classification scheme of $X$. translucens, rep-PCR genomic fingerprint profiles were obtained from all 33 isolates pathogenic on ornamental asparagus, as well as $61 \mathrm{X}$. translucens reference strains pathogenic to cereals and grasses. Amplified ribosomal gene restriction analysis (ARDRA) profiles were obtained from a subset of 42 strains. Rep-PCR genomic fingerprint profiles of all the strains were compared with a large Xanthomonas database using computerassisted pattern analysis (29). Rep-PCR genomic fingerprinting facilitated the characterization and discrimination of $X$. translucens, including the pathovars arrhenatheri, graminis, phlei, phleipratensis, poae, and cerealis, as well as the recently redefined $X$. translucens pv. undulosa and $X$. translucens pv. translucens groups (3). All 33 novel isolates from ornamental asparagus (A. virgatus) were identified as $X$. translucens pv. undulosa.

\section{MATERIALS AND METHODS}

Strains. Xanthomonas isolates used in this study are listed in Table 1. Isolates analyzed by ARDRA, in addition to those listed in Table 1 , are $X$. fragariae LMG 708T; $X$. hortorum pv. pelargonii LMG 7314*; X. populi LMG 5753; X. arboricola pvs.

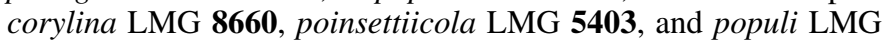
12141*; $X$. cassavae LMG 5264; $X$. bromi LMG 8267; $X$. cucurbitae LMG 8662; X. axonopodis pvs. begoniae LMG 7303*, vesicatoria LMG 910, axonopodis LMG 538t1T, phaseoli LMG 7455*, cajani LMG 7473, citri (A) LMG 682*, and dieffenbachiae LMG 7400; X. oryzae pv. oryzae LMG 5047T; $X$. vasicola pv. holcicola LMG 7416; $X$. campestris pvs. aberrans LMG 9037*, armoraciae LMG 535*, barbareae LMG 547*, campestris LMG 568T, incanae LMG 7490*, and raphani LMG 8134; $X$. hyacinthi LMG 8041; X. theicola LMG 8684*; X. sacchari LMG 471; and $X$. albilineans LMG 494T. An asterisk (*) indicates pathovar reference strain and $T$ indicates type strain. Strains in bold were included in the studies of Vauterin et al. (34) and Rademaker et al. (26). All strains have been used in Rademaker et al. (29).

Rep-PCR genomic fingerprinting. A worldwide collection of 61 reference strains composing all $10 X$. translucens pathovars as well as the 33 novel isolates from tree fern (Table 1) were subjected to rep-PCR genomic fingerprinting using primer sets corresponding to the BOX, ERIC, and REP elements (39). Isolation of total genomic DNA, rep-PCR, and electrophoresis conditions were as described before (25,27-29). From reference strains, duplicate fingerprints were generated. Moreover, two strains were analyzed (starting from cell samples) three times, eight strains twice, and the others once (Table 1).

ARDRA. Primers 49-68F TAACACATGCAAGTCGAACG and 1510-1492R AGTCGTAACAAGGTAACCG were used to amplify nearly full-length $16 \mathrm{~S}$ rRNA genes. PCR was performed in $50-\mu$ reaction mixtures containing $5 \mu \mathrm{l}$ of $10 \times$ PCR buffer $\left(500 \mathrm{mM} \mathrm{KCl}, 100 \mathrm{mM}\right.$ Tris- $\mathrm{HCl}\left[\mathrm{pH} 9.0\right.$ at $\left.25^{\circ} \mathrm{C}\right]$, and $1 \%$ Triton X-100) (Promega Corp.), $2.5 \mathrm{mM} \mathrm{MgCl}_{2}, 200 \mu \mathrm{M}$ each nucleotide, $1 \mu \mathrm{M}$ each primer, acetylated bovine serum albumin at $200 \mathrm{ng} / \mu \mathrm{l}, 3 \mathrm{U}$ of Taq polymerase, and $50 \mathrm{ng}$ of purified template DNA. The temperature profile was as follows: initial denaturation at $94^{\circ} \mathrm{C}$ for $3 \mathrm{~s}$; followed by 30 cycles of $92^{\circ} \mathrm{C}$ for $30 \mathrm{~s}, 53^{\circ} \mathrm{C}$ for $1 \mathrm{~min} 30 \mathrm{~s}$, and $72^{\circ} \mathrm{C}$ for $3 \mathrm{~min}$; with a final extension at $72^{\circ} \mathrm{C}$ for $7 \mathrm{~min}$. Aliquots $(15-\mu \mathrm{l})$ were incubated at $37^{\circ} \mathrm{C}$ for $4 \mathrm{~h}$ to overnight in $30-\mu$ l reaction mixtures with $5 \mathrm{U}$ of restriction endonucleases RsaI (New England Biolabs) in buffer NEB 1, or MspI (10 units) (New England Biolabs) and AluI (4 U) (New England Biolabs) in buffer NEB 2. These enzymes for the (double) digests were chosen to efficiently assess the expected diversity (20). The digests $(15 \mu \mathrm{l})$ were resolved by electrophoresis using $3.5 \%$ Metaphor in $1 \times$ Tris-borate EDTA (30) at $240 \mathrm{~V}$ for $15 \mathrm{~min}$, then $160 \mathrm{~V}$ for 3.5 to $4 \mathrm{~h}$, and then were stained with ethidium bromide. Molecular size marker V (Boehringer-Mannheim) was run at both sides and in the central lane of each gel.

Computer-assisted analysis of ARDRA profiles and repPCR genomic fingerprints. Gel images were digitized with a CCD videocamera and stored to disk as tagged image file format (TIFF) files. Gel images also were documented as Polaroid photographs, scanned with a Hewlett Packerd ScanJet 3C and Deskscan II v.2.2 software, and stored as TIFF files. The digitized images were converted, normalized using the molecular size marker $\mathrm{V}$ or the 1-kb size marker, respectively, and analyzed with GelCompar (version 4.1; Applied Maths, Kortrijk, Belgium) (37) as described $(25,28,29)$. The "rolling disk" background subtraction method was applied. The similarity between pairs of separate or linearly combined fingerprints was calculated using the product-moment correlation coefficient ( $r$ value) applied to the whole densitometric curves of the gel tracks $(11,28)$. The rep-PCR genomic fingerprints from $210 \mathrm{bp}$ to $7.5 \mathrm{~kb}$ were compared. SSU rDNARFLP profiles were compared using ranges from 45 to $740 \mathrm{bp}$. Cluster analysis of similarity matrices was performed using the unweighted pair group method using arithmetic averages (UPGMA) clustering technique. Fingerprint profiles were reconstructed as described $(25,28,29)$. The reproducibility was monitored by generating two independent rep-PCR genomic fingerprint profiles from each DNA sample, in separate PCR reactions and gels. Additionally, several independent DNA samples were obtained from some strains.

Bacteriophage tests. Bacterial isolates (Table 1) were grown in nutrient glucose broth for $24 \mathrm{~h}$. Subsequently, $200 \mu \mathrm{l}$ of the culture were added to $3 \mathrm{ml}$ of molten soft glycerol agar (SGA) (9) and spread over a nutrient agar plate. After the SGA solidified, one drop each of the four phage isolates $(17,18,21,23)$ was spotted on the SGA and plates were incubated at room temperature for $72 \mathrm{~h}$ before examination (9).

Plant pathogenicity tests on wheat and asparagus: mist inoculation method. The wheat cv. Florida 302 was selected for its known susceptibility to $X$. translucens (18). In all, 7 to 10 wheat seed were planted into $10-\mathrm{cm}$ pots $\left(450 \mathrm{~cm}^{3}\right)$ containing Vergo Container Mix A (Verlite Company, Tampa, FL) amended with Sierra mix (17N-6P-12K plus minor nutrients; Grace/Sierra, Milpitas, CA) at $1.5 \mathrm{~g} /$ pot. Wheat seedlings were inoculated after 3 weeks of growth (10 to $17 \mathrm{~cm}$ in height). A. virgatus rhizomes were planted into $15-\mathrm{cm}$ pots $\left(2,119 \mathrm{~cm}^{3}\right)$ containing the same soil mix and fertilizer ratio. Asparagus plants were inoculated only when new fronds were developing. Inoculum was prepared by growing strains for $24 \mathrm{~h}$ at $27 \pm 1{ }^{\circ} \mathrm{C}$ on Bacto nutrient agar amended with $5 \%$ sucrose. Bacteria were harvested from petri dishes, suspended in sterile saline $\left(\mathrm{NaCl}, 8.5 \mathrm{~g} \mathrm{liter}^{-1}\right)$, and spectrophotometrically adjusted (absorbance at $600 \mathrm{~nm}$ ) to an optical density of 0.3 , corresponding to $5 \times 10^{8} \mathrm{CFU} \mathrm{m}^{-1}$. Bacterial suspensions of reference and asparagus strains (Table 1) were applied to plants in each of three pots of each plant species. Suspensions were mist inoculated into clear polyethylene bags containing individual plant species, until the leaf surfaces of the plants were covered. Hand pump dispensers were utilized to produce a very fine aerosol mist that coated the leaves. The bags were removed from the plants after $24 \mathrm{~h}$. Inoculated plants were maintained in a glasshouse on mist benches, with mist generation above the plants once every $30 \mathrm{~min}$ for $15 \mathrm{~s}$ during daylight hours. Temperatures in the glasshouse were maintained between 18 and $32^{\circ} \mathrm{C}$ with a maximum lighting of $266 \mu \mathrm{mol} \mathrm{m} \mathrm{m}^{-2} \mathrm{~s}^{-1}$. Plants were examined for symptoms over a 4-week period. All inoculation experiments included six plants sprayed with sterile saline as a negative control. In addition, three strains of $X$. axonopodis pv. begoniae (ATCC 8718), X. hortorum pv. hederae (NCPPB 939), and X. campestris pv. zinniae (NCPPB 189) were inoculated to wheat, asparagus, 
TABLE 1. Strain information and results of rep-polymerase chain reaction (PCR) genomic fingerprinting, amplified ribosomal DNA restriction enzyme analysis (ARDRA), and phage and plant pathogenicity tests of isolates used in this study

\begin{tabular}{|c|c|c|c|c|c|c|c|c|c|c|c|}
\hline Strain no. ${ }^{\mathrm{a}}$ & Received as & Host & $\begin{array}{c}\text { Geographic } \\
\text { origin }\end{array}$ & $\mathrm{Fp}^{\mathrm{b}}$ & ARDRA $^{c}$ & Phage tests ${ }^{d}$ & $\begin{array}{l}\text { Wheat } \\
\mathrm{M}^{\mathrm{e}}\end{array}$ & $\begin{array}{l}\text { Asp } \\
M^{f}\end{array}$ & $\begin{array}{c}\text { Wheat } \\
\mathrm{F}^{\mathrm{g}}\end{array}$ & $\begin{array}{c}\text { Wheat } \\
\mathrm{S}^{\mathrm{h}}\end{array}$ & $\begin{array}{l}\text { Path } \\
\text { group }\end{array}$ \\
\hline \multicolumn{12}{|l|}{ X.t. undulosa } \\
\hline LMG 885 & X.t. undulosa & Triticum aestivum & Canada & 1 & nd & nd & nd & nd & nd & nd & nd \\
\hline LMG 886 & X.t. undulosa & Triticale & Canada & $1(2 \times)$ & nd & nd & + & + & nd & nd & nd \\
\hline LMG 888 & X.t. undulosa & Triticale & Canada & 1 & nd & nd & nd & nd & nd & nd & A \\
\hline LMG 892 & X.t. undulosa & Triticium turgidum & Canada & 1 & + & nd & nd & nd & nd & nd & A \\
\hline LMG 8283 & X.t. undulosa & Triticum aestivum & Canada & 1 & nd & nd & nd & nd & nd & nd & nd \\
\hline LMG 887 & X.t. cerealis & Agropyron repens & Canada & 1 & nd & nd & nd & nd & nd & nd & nd \\
\hline LMG 890 & X.t. cerealis & Triticum aestivum & Canada & 1 & nd & nd & nd & nd & nd & nd & nd \\
\hline LMG 7392 & X.t. cerealis & Secale cereale & United States & $1(2 \times)$ & nd & NNNNNNNN & + & + & - & - & nd \\
\hline LMG 877 & X.t. secalis & Secale cereale & Canada & 1 & nd & РPPPPPPP & + & + & + & + & nd \\
\hline LMG 883 & X.t. secalis & Secale cereale & Canada & 1 & + & nd & nd & nd & nd & nd & A \\
\hline LMG 7445 & X.t. secalis & Secale cereale & Canada & 1 & nd & VWNWNNNNN & + & + & + & + & nd \\
\hline LMG 7507 & X.t. secalis & Secale cereale & Canada & 1 & nd & nd & nd & nd & nd & nd & nd \\
\hline LMG 878 & X.t. translucens & Triticum aestivum & Kenya & 1 & nd & VWNWNNNNN & + & - & + & + & nd \\
\hline LMG 5259 & X.t.translucens & Secale cereale & United States & 1 & nd & nd & nd & nd & nd & nd & A \\
\hline LMG 5260 & X.t. translucens & Secale cereale & United States & $1(2 x)$ & nd & nd & nd & nd & nd & nd & nd \\
\hline LMG 5261 & X.t. translucens & Secale cereale & United States & 1 & nd & NNNNNNNN & + & + & - & - & nd \\
\hline LMG 5262 & X.t. translucens & Triticale & United States & 1 & nd & nd & nd & nd & nd & nd & nd \\
\hline LMG 5263 & X.t. translucens & Triticum aestivum & United States & 1 & nd & NNNNNNNN & + & + & - & - & nd \\
\hline ATCC 10771 & X.t. translucens & Triticum aestivum & Unknown & $1^{*}$ & nd & PPPPPWNN & + & + & - & - & nd \\
\hline X1553 & Xanthomonas sp. & Asparagus virgatus & Florida & 1 & + & NNNN & + & + & nd & nd & nd \\
\hline $\mathrm{X} 1554$ & Xanthomonas sp. & Asparagus virgatus & Florida & 1 & + & NNNNNNNN & + & + & - & - & nd \\
\hline $\mathrm{X} 1555$ & Xanthomonas sp. & Asparagus virgatus & Florida & 1 & + & VWNNN & + & + & nd & nd & nd \\
\hline X1556 & Xanthomonas sp. & Asparagus virgatus & Florida & 1 & + & NNNN & + & + & nd & nd & nd \\
\hline X1557 & Xanthomonas sp. & Asparagus virgatus & Florida & 1 & + & NNNN & + & + & nd & nd & nd \\
\hline $\mathrm{X} 1558$ & Xanthomonas sp. & Asparagus virgatus & Florida & 1 & nd & NNNN & + & + & nd & nd & nd \\
\hline X1571 & Xanthomonas sp. & Asparagus virgatus & Florida & 1 & + & VWNNN & + & + & nd & nd & nd \\
\hline $\mathrm{X} 1572$ & Xanthomonas sp. & Asparagus virgatus & Florida & 1 & + & WNNN & + & + & nd & nd & nd \\
\hline X1573 & Xanthomonas sp. & Asparagus virgatus & Florida & 1 & + & WNNN & + & + & nd & nd & nd \\
\hline X1574 & Xanthomonas sp. & Asparagus virgatus & Florida & 1 & + & WNNN & + & + & nd & nd & nd \\
\hline X1575 & Xanthomonas sp. & Asparagus virgatus & Florida & 1 & + & NNNN & + & + & nd & nd & nd \\
\hline X1576 & Xanthomonas sp. & Asparagus virgatus & Florida & 1 & + & NNNN & + & + & nd & nd & nd \\
\hline X1577 & Xanthomonas sp. & Asparagus virgatus & Florida & 1 & + & NNNN & + & + & nd & nd & nd \\
\hline $\mathrm{X} 1578$ & Xanthomonas sp. & Asparagus virgatus & Florida & 1 & + & NNNNNNNN & + & + & - & - & nd \\
\hline X1579 & Xanthomonas sp. & Asparagus virgatus & Florida & 1 & + & WNNN & + & + & nd & nd & nd \\
\hline $\mathrm{X} 1580$ & Xanthomonas sp. & Asparagus virgatus & Florida & 1 & nd & NNNN & + & + & nd & nd & nd \\
\hline $\mathrm{X} 1581$ & Xanthomonas sp. & Asparagus virgatus & Florida & 1 & + & NNNN & + & + & nd & nd & nd \\
\hline X1582 & Xanthomonas sp. & Asparagus virgatus & Florida & 1 & + & VWNNN & + & + & nd & nd & nd \\
\hline $\mathrm{X} 1583$ & Xanthomonas sp. & Asparagus virgatus & Florida & 1 & + & NNNNNNNN & + & + & - & - & nd \\
\hline X1584 & Xanthomonas sp. & Asparagus virgatus & Florida & 1 & + & VWNNN & + & + & nd & nd & nd \\
\hline X1585 & Xanthomonas sp. & Asparagus virgatus & Florida & 1 & + & WNNN & + & + & nd & nd & nd \\
\hline X1586 & Xanthomonas sp. & Asparagus virgatus & Florida & 1 & + & VWNNN & + & + & nd & nd & nd \\
\hline X1587 & Xanthomonas sp. & Asparagus virgatus & Florida & 1 & nd & NNNN & + & + & nd & nd & nd \\
\hline $\mathrm{X} 1588$ & Xanthomonas sp. & Asparagus virgatus & Florida & 1 & + & NNNNNNNN & + & + & - & - & nd \\
\hline X1589 & Xanthomonas sp. & Asparagus virgatus & Florida & 1 & + & VWNNN & + & + & nd & nd & nd \\
\hline X1592 & Xanthomonas sp. & Asparagus virgatus & Florida & 1 & + & VWNNN & + & + & nd & nd & nd \\
\hline X1593 & Xanthomonas sp. & Asparagus virgatus & Florida & 1 & + & NNNN & + & + & nd & nd & nd \\
\hline X1594 & Xanthomonas sp. & Asparagus virgatus & Florida & 1 & nd & NNNNNNNN & + & + & - & - & nd \\
\hline X1595 & Xanthomonas sp. & Asparagus virgatus & Florida & 1 & + & NNNN & + & + & nd & nd & nd \\
\hline X1596 & Xanthomonas sp. & Asparagus virgatus & Florida & 1 & + & VWNNN & + & + & nd & nd & nd \\
\hline X1597 & Xanthomonas sp. & Asparagus virgatus & Florida & 1 & + & NNNNNNNN & + & + & - & - & nd \\
\hline X1598 & Xanthomonas sp. & Asparagus virgatus & Florida & 1 & nd & NNNNNNNN & + & + & - & - & nd \\
\hline X1599 & Xanthomonas sp. & Asparagus virgatus & Florida & 1 & nd & VWNNN & + & + & nd & nd & nd \\
\hline \multicolumn{12}{|l|}{ X.t. translucens } \\
\hline LMG 875 & X.t. translucens & Hordeum vulgare & Canada & 3 & + & nd & nd & nd & nd & nd & nd \\
\hline LMG 876 & X.t. translucens & Hordeum vulgare & United States & 3 & + & nd & nd & nd & nd & nd & nd \\
\hline \multirow[t]{2}{*}{ ATCC 9002} & X.t. translucens & Unknown & Unknown & $5^{*}$ & nd & NNNNNNNN & + & - & - & - & nd \\
\hline & & & & & & & & & \multicolumn{3}{|c|}{ (continued on next page) } \\
\hline
\end{tabular}

${ }^{a}$ Xanthomonas translucens (X.t.) strain number: strains were obtained from LMG, culture collection of the Laboratorium voor Microbiologie, Universiteit Gent, Gent, Belgium; ATCC, the American Type Culture Collection, Manassas, VA; or NCPPB, National Collection of Plant Pathogenic Bacteria CSL. York numbers starting with $\mathrm{X}$ from David Norman, others from Robert Forster; numbers in bold were used in a supporting study by Bragard et al. (3).

${ }^{\mathrm{b}} \mathrm{Fp}=$ cluster number of combined BOX-, ERIC-, and REP-PCR genomic fingerprint profiles from Figure 1 and matching the numbers used in a supporting study $(3) ; *$ data not shown; numbers in parentheses refers to number of times ( $2 \times$ to $3 \times$ ) the strains were independently analyzed; absence of a number in parentheses indicates a single analysis; $\mathrm{smc}=$ single member cluster; $\mathrm{nd}=$ not determined.

$c_{+}+=$X. translucens-specific MspI/AluI ARDRA profile was obtained characterized by the absence of an $\approx 130$ - and $\approx 165$-bp fragment; nd = not determined.

${ }^{\mathrm{d}} \mathrm{P}=$ strong; $\mathrm{W}=$ weak; $\mathrm{VW}=$ very weak reaction; $\mathrm{N}=$ no reaction with phages $(17,18,21,23)$ or $(17,17,18,18,21,21,23,23)$ specific for $X$. translucens pv. translucens; nd $=$ not determined.

e Plant pathogenicity tests on wheat using mist inoculation method.

${ }^{\mathrm{f}}$ Plant pathogenicity tests on asparagus using mist inoculation method.

g Plant pathogenicity tests on wheat using foil cap method.

${ }^{\mathrm{h}}$ Plant pathogenicity tests on wheat using syringe injection method.

${ }^{i}$ Pathogenicity groups as reported by Bragard et al. (3).

j All other reference strains were pathogenic to their original host; X. axonopodis (X.a.), X. hortorum (X.h.), and X. campestris (X.c.). 
and their original host. When symptoms developed on plants, bacteria were reisolated and presumptively identified as xanthomonads with the following tests: pigmentation on yeast dextrose calcium carbonate medium (YDC) (40); production of poly- $\beta$ hydroxybutyrate on Nile Blue medium (NB) (24); fluorescence on King's medium B (KMB) (14); starch hydrolysis on cellobiose starch medium (CS) (21); gram, oxidase, and catalase reactions, oxidation-fermentation of glucose; and phenotype on motility agar following protocols of Suslow et al. (32) and MacFaddin (16).

Plant pathogenicity tests on wheat: foil cap method. Inoculum was prepared by growing reference and asparagus strains (Table 1) for 3 days at $30^{\circ} \mathrm{C}$ on YDC. Two loops of bacteria were harvested from petri dishes and suspended in $10 \mathrm{ml}$ of sterile saline $\left(\mathrm{NaCl}, 8.5 \mathrm{~g} \mathrm{liter}^{-1}\right)$, resulting in suspensions of $\approx 6 \times$ $10^{8}$ to $6 \times 10^{9} \mathrm{CFU} \mathrm{ml^{-1 }}$. Bacterial suspension $(3 \mathrm{ml})$ of each

TABLE 1. (Continued from preceding page)

\begin{tabular}{|c|c|c|c|c|c|c|c|c|c|c|c|}
\hline Strain no. ${ }^{\mathrm{a}}$ & Received as & Host & $\begin{array}{c}\text { Geographic } \\
\text { origin }\end{array}$ & $\mathrm{Fp}^{\mathrm{b}}$ & $\mathrm{ARDRA}^{\mathrm{c}}$ & Phage tests ${ }^{\mathrm{d}}$ & $\begin{array}{c}\text { Wheat } \\
\mathrm{M}^{\mathrm{e}}\end{array}$ & $\begin{array}{l}\text { Asp } \\
M^{f}\end{array}$ & $\begin{array}{c}\text { Wheat } \\
\mathrm{Fg}^{\mathrm{g}}\end{array}$ & $\begin{array}{c}\text { Wheat } \\
\mathrm{S}^{\mathrm{h}}\end{array}$ & $\begin{array}{l}\text { Path } \\
\text { group }^{\mathrm{i}}\end{array}$ \\
\hline \multicolumn{12}{|l|}{ X.t. translucens } \\
\hline ATCC 10770 & X.t. translucens & Hordeum vulgare & Unknown & $5 *$ & nd & NNNNNNNN & + & - & - & - & nd \\
\hline ATCC 10731 & X.t. translucens & Hordeum vulgare & Unknown & $2 *$ & nd & NNNNNNNN & + & - & - & - & nd \\
\hline LMG 891 & X.t. cerealis & Triticum turgidum & Canada & 5 & nd & nd & nd & nd & nd & nd & nd \\
\hline LMG 737 & X.t. hordei & Hordeum vulgare & India & $5(2 \times)$ & nd & nd & nd & nd & nd & nd & $\mathrm{B}$ \\
\hline LMG 884 & X.t. hordei & Hordeum vulgare & Canada & 2 & nd & nd & nd & nd & nd & nd & nd \\
\hline LMG 889 & X.t. hordei & Hordeum vulgare & Canada & 2 & nd & NNNNNNNN & + & - & - & - & nd \\
\hline LMG 8279 & X.t. hordei & Hordeum vulgare & New Zealand & $2(3 \times)$ & + & nd & nd & nd & nd & nd & $\mathrm{B}$ \\
\hline LMG 882 & X.t. hordei & Hordeum vulgare & Canada & 3 & + & nd & nd & nd & nd & nd & B \\
\hline LMG 8280 & X.t. hordei & Hordeum vulgare & New Zealand & 5 & nd & NNNNNNNN & + & + & - & - & nd \\
\hline LMG 8281 & X.t. hordei & Hordeum vulgare & India & 5 & nd & NWVWPWWNN & + & - & - & - & nd \\
\hline LMG 8282 & X.t. undulosa & Triticum aestivum & Canada & 5 & nd & NNNNNNNN & + & - & - & - & nd \\
\hline \multicolumn{12}{|l|}{ X.t. cerealis } \\
\hline LMG 679 & X.t. cerealis & Bromus inermis & United States & $6(3 \times)$ & + & NNNNNNNN & + & + & - & - & A \\
\hline LMG 880 & X.t. cerealis & Hordeum vulgare & Canada & $6(2 \times)$ & + & PPPPWWNN & + & + & + & - & A \\
\hline LMG 881 & X.t. cerealis & Bromus inermis & Canada & 6 & nd & NNNNNNNN & + & + & - & - & nd \\
\hline LMG 7393 & X.t. cerealis & Hordeum vulgare & United States & 6 & nd & NNNNNNNN & + & - & - & - & nd \\
\hline \multicolumn{12}{|l|}{ X.t.poae } \\
\hline LMG 594 & X.t. poae & Poa trivialis & Switzerland & 7 & nd & nd & nd & nd & nd & nd & $\mathrm{C}$ \\
\hline LMG 728 & X.t. poae & Poa trivialis & Switzerland & 7 & + & nd & nd & nd & nd & nd & $\mathrm{C}$ \\
\hline ATCC 33804 & X.t.poae & Poa trivialis & Switzerland & $7 *$ & nd & NNNNNNNN & + & + & - & - & nd \\
\hline \multicolumn{12}{|l|}{ X.t.phlei } \\
\hline LMG 716 & X.t.phlei & Phleum pratense & Belgium & 9 & nd & nd & nd & nd & nd & nd & nd \\
\hline LMG 718 & X.t.phlei & Phleum pratense & Belgium & 9 & + & NNNNNNNN & + & - & - & - & nd \\
\hline LMG 719 & X.t.phlei & Phleum pratense & Belgium & 9 & nd & nd & nd & nd & nd & nd & nd \\
\hline LMG 720 & X.t.phlei & Phleum pratense & Belgium & $9(2 \times)$ & nd & nd & nd & nd & nd & nd & nd \\
\hline LMG 723 & X.t.phlei & Phleum pratense & Belgium & 9 & nd & nd & nd & nd & nd & nd & nd \\
\hline LMG 730 & X.t.phlei & Phleum pratense & Norway & 9 & + & nd & nd & nd & nd & nd & nd \\
\hline \multicolumn{12}{|c|}{ X.t. phleipratensis } \\
\hline LMG 843 & X.t. phleipratensis & Phleum pratense & United States & 9 & + & nd & nd & nd & nd & nd & $\mathrm{C}$ \\
\hline \multicolumn{12}{|c|}{ X.t. arrhenatheri } \\
\hline LMG 590 & X.t. arrhenatheri & Arrhenatherum elatius & Switzerland & 10 & nd & nd & nd & nd & nd & nd & nd \\
\hline LMG 591 & X.t. arrhenatheri & Arrhenatherum elatius & Switzerland & 10 & nd & nd & nd & nd & nd & nd & nd \\
\hline LMG 594 & X.t. arrhenatheri & Poa trivialis & Switzerland & 10 & nd & nd & nd & nd & nd & nd & nd \\
\hline LMG 727 & X.t. arrhenatheri & Arrhenatherum elatius & Switzerland & $10(2 \times)$ & + & nd & nd & nd & nd & nd & nd \\
\hline LMG 7384 & X.t. arrhenatheri & Arrhenatherum elatius & Switzerland & 10 & nd & nd & nd & nd & nd & nd & nd \\
\hline ATCC 33803 & X.t. arrhenatheri & Arrhenatherum elatius & Switzerland & $9 *$ & nd & NNNNNNNN & - & - & - & - & nd \\
\hline \multicolumn{12}{|l|}{ X.t. graminis } \\
\hline LMG 587 & X.t. graminis & Lolium sp. & The Netherlands & 11 & nd & nd & nd & nd & nd & nd & nd \\
\hline LMG 589 & X.t. graminis & Dactylis glomerata & Switzerland & 11 & nd & nd & nd & nd & nd & nd & nd \\
\hline LMG 595 & X.t. graminis & Dactylis glomerata & Norway & 11 & nd & nd & nd & nd & nd & nd & nd \\
\hline LMG 596 & X.t. graminis & Lolium multiflorum & Belgium & 11 & nd & nd & nd & nd & nd & nd & nd \\
\hline LMG 600 & X.t. graminis & Lolium perenne & Belgium & 11 & nd & nd & nd & nd & nd & nd & nd \\
\hline LMG 713 & X.t. graminis & Lolium perenne & Belgium & 11 & nd & nd & nd & nd & nd & nd & nd \\
\hline LMG 714 & X.t. graminis & Lolium perenne & Belgium & 11 & nd & nd & nd & nd & nd & nd & nd \\
\hline LMG 721 & X.t. graminis & Lolium perenne & Belgium & 11 & nd & nd & nd & nd & nd & nd & nd \\
\hline LMG 726 & X.t. graminis & Dactylis glomerata & Switzerland & $11(2 \times)$ & + & nd & nd & nd & nd & nd & nd \\
\hline LMG 729 & X.t. graminis & Lolium multiflorum & Switzerland & 11 & nd & nd & nd & nd & nd & nd & nd \\
\hline LMG 7406 & X.t. graminis & Dactylis glomerata & Switzerland & 11 & nd & nd & nd & nd & nd & nd & nd \\
\hline LMG 7407 & X.t. graminis & Lolium multiflorum & Switzerland & 11 & nd & nd & nd & nd & nd & nd & nd \\
\hline LMG 10380 & X.t. graminis & Lolium multiflorum & Unknown & 11 & nd & NNNNNNNN & + & - & - & - & nd \\
\hline \multicolumn{12}{|c|}{ Other X. translucens } \\
\hline LMG 879 & X.t. hordei & Dactylis glomerata & Japan & smc & + & nd & nd & nd & nd & nd & B \\
\hline $215 \mathrm{~A}$ & X.c. translucens & Triticum aestivum & Idaho & nd & nd & PPPN & nd & nd & + & nd & nd \\
\hline 399B & X.c. translucens & Triticum aestivum & Idaho & nd & nd & PPPN & nd & nd & + & - & nd \\
\hline $400 \mathrm{~A}$ & X.c. translucens & Hordeum vulgare & Idaho & nd & nd & NNNN & nd & nd & - & - & nd \\
\hline $406 \mathrm{~A}$ & X.c. translucens & Hordeum vulgare & Colorado & nd & nd & NNNN & nd & nd & - & - & nd \\
\hline $\mathrm{X} 1650$ & X.t. secalis & Sesamum indicum & India & nd & nd & NNNNNNNN & + & - & - & - & nd \\
\hline \multicolumn{12}{|l|}{ Other strains ${ }^{\mathrm{j}}$} \\
\hline ATCC 8718 & X.a. begoniae & Tuberous begonia & Unknown & nd & nd & NNNNNNNN & nd & nd & nd & nd & nd \\
\hline NCPPB 939 & X.h. hederae & Hedera helix & United States & nd & nd & NNNNNNNN & nd & nd & nd & nd & nd \\
\hline NCPPB 189 & X.c. zinniae & Zinnia sp. & Zimbabwe & nd & nd & NNNNNNNN & nd & nd & nd & nd & nd \\
\hline
\end{tabular}


strain (reference and asparagus strains, Table 1) was placed in foil caps lined with absorbent cotton. Caps containing inoculum were placed over the cut leaves. The caps provide a humid environment that promotes infection.

Leaves of three plants (cv. Whitebird) at the three-leaf stage were cut with a razor blade $\approx 5 \mathrm{~cm}$ above the soil line. Foil caps immediately were placed over the cut leaves of two plants and left there for $24 \mathrm{~h}$. The third plant served as a negative control. The plants were grown in $11-\mathrm{cm}$ plastic pots filled with pasteurized compost mix. Temperatures averaged $27^{\circ} \mathrm{C}$ in the daytime to $11^{\circ} \mathrm{C}$ at night. Plants were examined 12 days after inoculation.

Plant pathogenicity tests on wheat: syringe injection method. Inoculum was prepared by growing reference and asparagus strains (Table 1) for 2 days at $30^{\circ} \mathrm{C}$ on YDC. One loop of bacteria was harvested from petri plates and suspended in $9 \mathrm{ml}$ of sterile saline $\left(\mathrm{NaCl}, 8.5 \mathrm{~g} \mathrm{liter}^{-1}\right)$, resulting in suspensions of $\approx 1 \times$ $10^{9} \mathrm{CFU} \mathrm{ml}{ }^{-1}$. Then, $\approx 20 \mu \mathrm{l}$ of the bacterial suspension of each strain (reference and asparagus strains, Table 1) was injected into the base of three wheat plants (cv. Whitebird) at the three-leaf stage using an insulin syringe with a 26 -gauge needle. The pots immediately were placed in clear plastic bags in the greenhouse for 2 days, after which the bags were removed. The plants were grown in 11-cm plastic pots filled with pasteurized compost mix. Temperatures averaged $27^{\circ} \mathrm{C}$ in the daytime and $11^{\circ} \mathrm{C}$ at night. Plants were examined 19 days after inoculation. Reisolations were carried out using modified Xanthomonas translucens seed (XTS) agar (10) from plants exhibiting watersoaking and necrosis. The isolated cultures were plated onto YDC to test for normal growth and morphology.

\section{RESULTS}

Rep-PCR genomic fingerprinting and analysis. Highly complex rep-PCR genomic fingerprint profiles, including several strain-specific profiles, were obtained from 33 novel asparagus strains and $61 X$. translucens strains. All asparagus strains were analyzed as independent samples twice; eight other Xanthomonas strains were analyzed twice, and two were analyzed three times, resulting in a database of 630 fingerprint profiles (Table 1; Fig. 1). The BOX, ERIC, and REP fingerprints were linearly combined and compared using the product moment correlation coefficient submitted to UPGMA cluster analysis (Fig. 1). Strains received as pathovars graminis, poae, and arrhenatheri grouped in individual pathovar groups (clusters 11, 7, and 10 respectively) and strains from pathovars phlei and phleipratensis grouped together in cluster 9 (Fig. 1). The X. translucens pv. graminis-specific cluster 11 comprised 28 profiles of 13 strains (Table 1; Fig. 1). Strains LMG 594 and 728 formed a pv. poae-specific cluster (cluster 7) (Table 1; Fig. 1), each exhibiting strain-specific polymorphisms. Ten profiles of arrhenatheri strains formed a single and pathovarspecific coherent cluster (cluster 10) (Table 1; Fig. 1). Twelve profiles of five pv. phlei strains formed a coherent cluster (cluster 9) (Table 1; Fig. 1) and showed similarity to LMG 730 and 843, received as pvs. phlei and phleipratensis, respectively.

Four strains received as $X$. translucens pv. cerealis grouped together in a separate, single cluster (cluster 6) (Table 1; Fig. 1). Strains received as pvs. hordei, secalis, translucens, and undulosa and the remainder of the pv. cerealis isolates grouped together, which was not in agreement with the original pathovar classification as received. Four separate subclusters (clusters 1, 2, 3, and 5) were recognized (Table 1; Fig. 1). The cluster referred to as cluster 1 included strains received as $X$. translucens pvs. secalis, undulosa, cerealis, and translucens (Table 1; Fig. 1). The other three clusters (referred to as clusters 2, 3, and 5) comprised strains predominantly received as $X$. translucens pv. hordei and did not include any $X$. translucens pv. secalis strains. Cluster 2 comprised strains received as pv. hordei, cluster 3 comprised strains received as pvs. hordei and translucens, and cluster 5 comprised strains received as pvs. hordei, cerealis, and undulosa (Table 1; Fig. 1). One additional strain, received as $X$. translucens pv. hordei LMG 879, constituted a single-member cluster (Fig. 1).

The differentiation of strains documented in this report supports the results of Bragard et al. (3), who used 11 equivalent reference strains (Table 1) and AFLP analysis to study diversity and pathogenicity within the $X$. translucens group. The clusters described by Bragard and co-workers (and the numbers used to refer to the clusters [i.e., 1, 2, 3, 5, 6, 7, 9, 10, and 11]) correspond directly to the clusters observed in the current study.

All 33 novel isolates pathogenic to ornamental asparagus (A. virgatus) grouped at a high similarity in cluster 1 (Fig. 1). These isolates are the first-described bacterial pathogens of asparagus (22); all hosts of $X$. translucens pathovars described previously consisted of only cereals and grasses.

When strains were compared using their BOX-, ERIC-, or REP-PCR fingerprints separately, equivalent results were obtained, with the notable difference that the species $X$. translucens usually was not assembled in one single cluster in the large database (29). Abridged clusters of BOX-PCR genomic fingerprint profiles show the genomic groups observed (Fig. 2). With the separate and combined fingerprints, there was a low similarity between rep-PCR genomic fingerprints of the $X$. translucens strains (including those from asparagus) and profiles of other Xanthomonas strains, including pvs. dieffenbachiae and vitians (29; data not shown).

ARDRA. ARDRA (20) of the SSU ribosomal gene using a single and a double digest was used for further characterization of selected $X$. translucens isolates, including the novel asparagus isolates (Table 1) and 29 additional Xanthomonas reference strains composing 16 species. SSU ribosomal gene fingerprinting using $R s a \mathrm{I}$ yielded one fingerprint profile for Xanthomonas strains from all 16 species analyzed in the current study. Moreover, a unique, $X$. translucens-specific $M s p \mathrm{I} / A l u \mathrm{I}$ ARDRA fingerprint profile was observed that was characterized by the absence of an $\approx 130$ - and $\approx 165$-bp fragment, indicated by a positive score in Table $1 . \mathrm{Msp} \mathrm{I} /$ AluI ARDRA profiles of $X$. albilineans, $X$. hyacinthi, and $X$. theicola reference strains also missed the $\approx 165$-bp fragment but always showed the $\approx 130$-bp fragment. Support for the identification of the novel asparagus isolates as $X$. translucens was obtained using ARDRA because the Xanthomonas spp.-specific RsaI and $X$. translucens-specific $M s p \mathrm{I} / A l u \mathrm{I}$ ARDRA fingerprint profile were observed for these isolates (Table 1; data not shown).

Bacteriophage testing. All 33 isolates from ornamental asparagus were analyzed for sensitivity to four bacteriophage isolates, referred to as $17,18,21$, and 23 , that originally were isolated from wheat (Triticum aestivum $\mathrm{L}$.) pathogens (9). Weak (5/33) to very weak (9/33) reactions occurred with phage 17 and no reaction with phages 18, 21, and 23 (Table 1). Reference strains LMG 8281, LMG 7445, and LMG 878 gave only weak and very weak reactions, whereas only strains LMG 877, LMG 880, and ATCC 10771 and control strains $215 \mathrm{~A}$ and $399 \mathrm{~B}$ showed positive reactions.

Plant pathogenicity tests on wheat and asparagus using mist inoculation. All 33 novel asparagus isolates and 24 reference strains received as belonging to $X$. translucens pvs. arrhenatheri, graminis, phlei, poae, hordei, secalis, translucens, undulosa, and cerealis were tested for pathogenicity on wheat and asparagus tree fern (Table 1). All 33 novel asparagus isolates and 23 of the 24 reference strains infected wheat plants in Florida. Small water-soaked, chlorotic spots were observed on leaves of infected plants. Only X. translucens pv. arrhenatheri strain ATCC 33803, originally isolated from oat grass (Arrhenatherum elatius), did not infect wheat. The most severe symptoms were observed with some of the original wheat isolates. Water-soaked lesions also formed on asparagus stems as expected with all 33 novel asparagus isolates and an additional 12 of the 24 reference $X$. translucens strains tested (Table 1). The reference strains that 


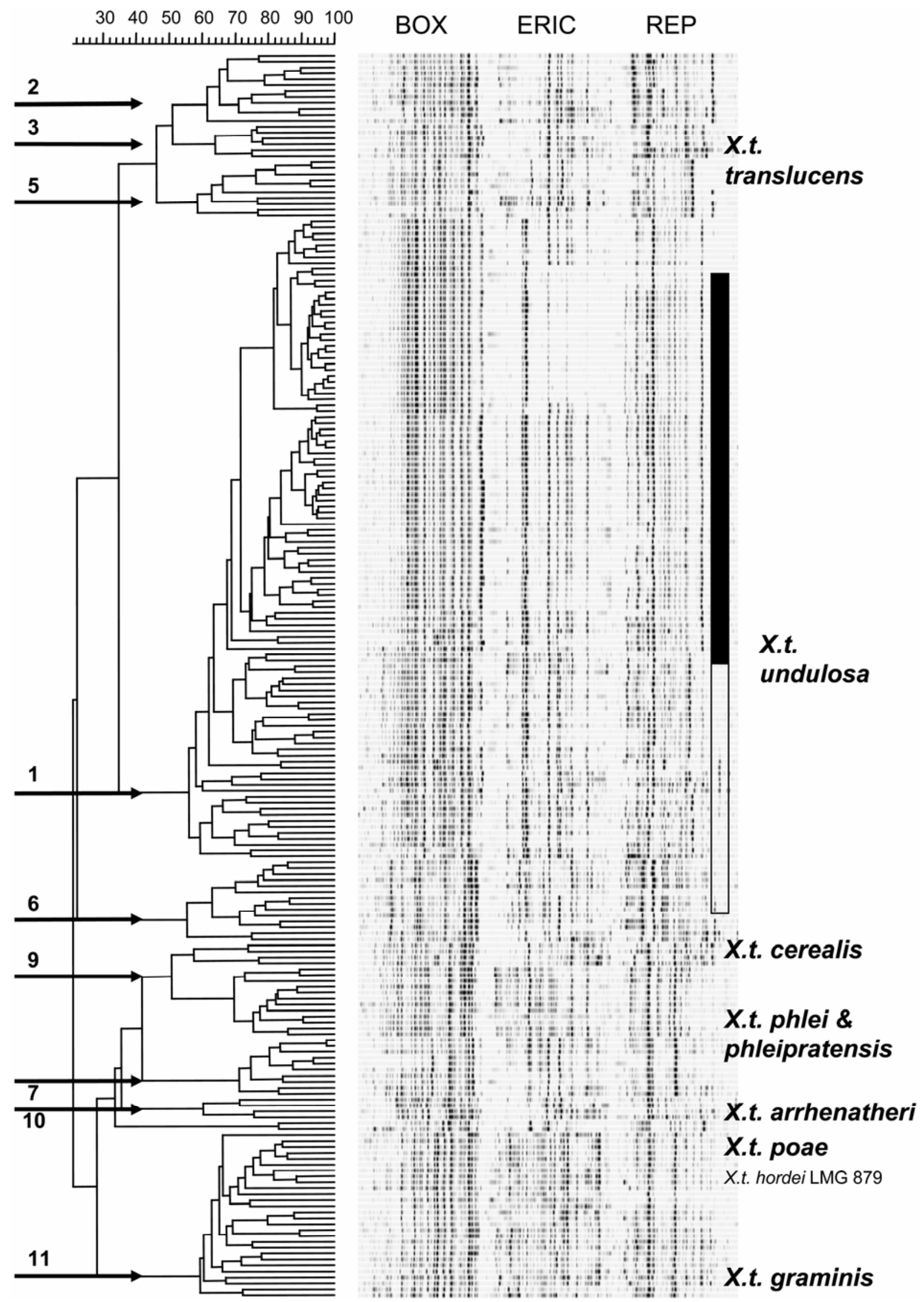

Fig. 1. Unweighted pair group method using arithmetic averages/product moment cluster analysis of combined BOX-, ERIC-, and REP-polymerase chain reaction genomic fingerprints of Xanthomonas translucens reference strains and novel isolates pathogenic on ornamental asparagus. Clusters X. translucens (X.t.) undulosa, number 1; X.t. translucens, numbers 2, 3, and 5; X.t. cerealis, number 6; X.t. poae, number 7; X.t. phlei and phleipratensis, number 9; X.t. arrhenatheri, number 10; and X.t. graminis, number 11 correspond directly to the clusters described by Bragard et al. (3), after amplified fragment length polymorphism genomic fingerprint analysis. Black bars indicate novel asparagus pathogens, the open bar marks the additional strains classified as X. translucens pv. undulosa. Further explanation provided in text. Similarity coefficient is shown as percentage. 
also affect asparagus include seven strains of $X$. translucens pv. undulosa, one of X. translucens pv. translucens, three of X. translucens pv. cerealis, and one of X. translucens pv. poae (Table 1). The strains could not be distinguished by disease symptomology; however, the most severe symptoms usually were observed on host of origin. Reisolations from asparagus, wheat, and control plants were done for each strain inoculated. No symptoms developed on plants inoculated with the saline control. Water-soaked lesions developed on the original hosts of the begoniae, hederae, and zinniae strains; however, these strains did not produce disease symptoms on either wheat or asparagus (data not shown). All reisolated strains from wheat, asparagus, and controls grew readily on YDC medium, forming large, round, butyrous, and bright yellow colonies within $48 \mathrm{~h}$. All reisolated strains were gramstaining negative, oxidase negative, catalase positive, motile, did not hydrolyze starch, were nonfluorescent, strictly aerobic, and did not form poly- $\beta$-hydroxybutyrate.

Plant pathogenicity tests on wheat using foil cap and syringe inoculation. Positive pathogenicity reactions on wheat inoculated using both foil cap and syringe methods were observed for $X$. translucens pv. secalis strains LMG 7445 and LMG 877 and $X$. translucens pv. translucens strain LMG 878, which grouped together in rep-PCR genomic fingerprinting cluster 1 (Table 1; Fig. 1). Additionally, a positive pathogenicity reaction on wheat using the foil cap method was observed only for

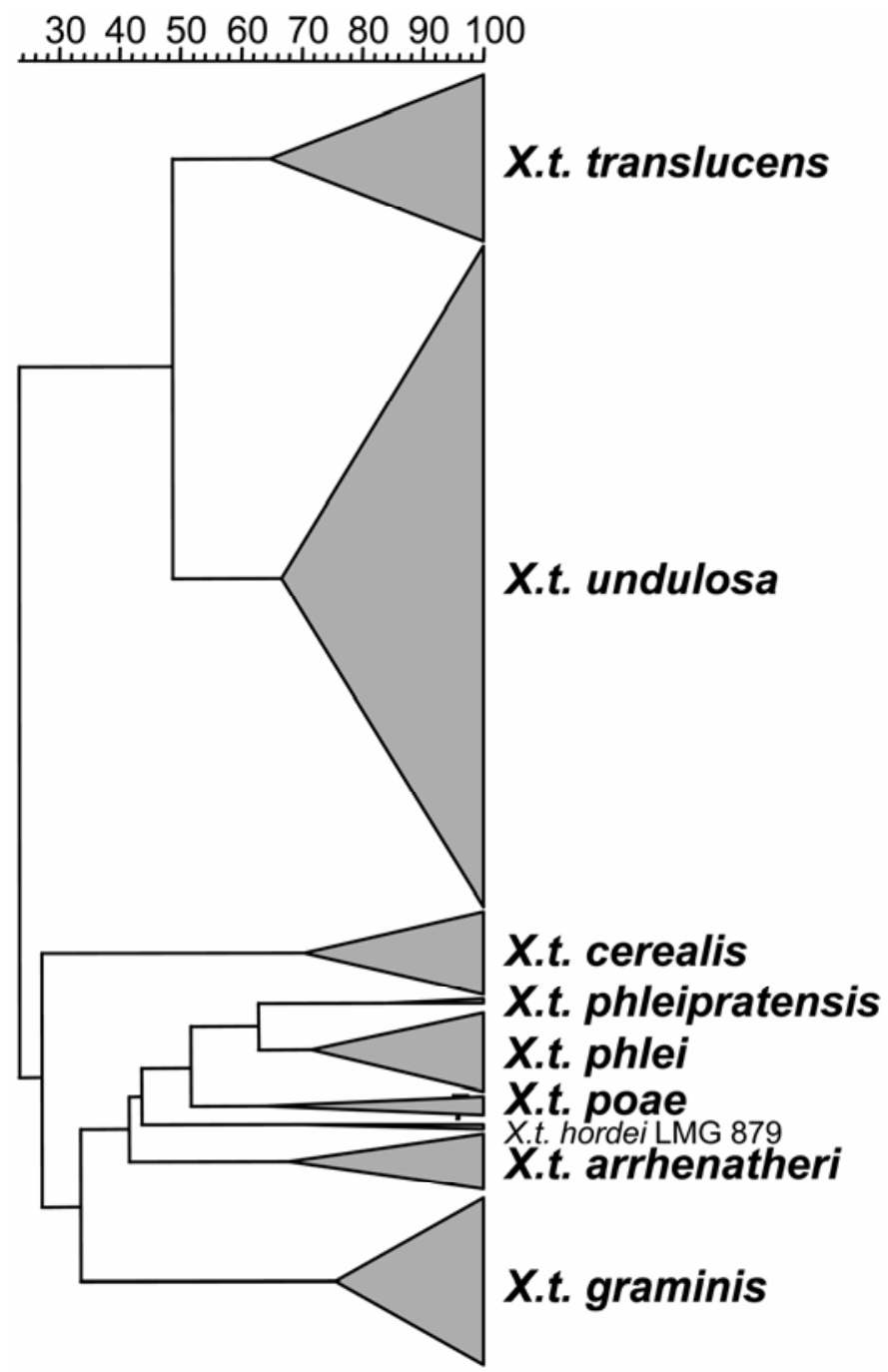

Fig. 2. Unweighted pair group method using arithmetic averages/product moment cluster analysis of BOX-polymerase chain reaction genomic fingerprints of Xanthomonas translucens (X.t.) reference strains and novel isolates pathogenic on ornamental asparagus (Fig. 1; Table 1).
$X$. translucens pv. serealis strain LMG 880, which grouped in repPCR genomic fingerprinting cluster 6 (Table 1; Fig. 1).

\section{DISCUSSION}

Xanthomonas strains pathogenic on grasses and cereals comprise many different pathovars and strains. However, recently, all strains examined were found to belong to one large cluster (X. translucens) in a database of over 300 Xanthomonas isolates (29). This report details the subspecific structure of $X$. translucens more thoroughly. Rep-PCR genomic fingerprinting and computerassisted analysis permitted the discrimination of the $X$. translucens pvs. arrhenatheri, graminis, phlei, phleipratensis, and poae and part of $X$. translucens $\mathrm{pv}$. cerealis. In a previous study, a high correlation between rep-PCR genomic fingerprinting and DNADNA hybridization studies was described, highlighting the taxonomic utility of the method (26). In the present study, pvs. hordei, secalis, translucens, and undulosa and another part of the X.translucens pv. cerealis isolates clustered together, but were not grouped by their pathovar classification as received. However, they formed two coherent and meaningful groups, one of which contained three subclusters. These clusters are supported by their direct correspondence to the clusters reported by Bragard et al. (3), who used 11 equivalent reference strains in AFLP genomic fingerprint analysis and pathogenicity studies (for convenience, corresponding groups are assigned the corresponding characters: $1,2,3,5,6,7,9,10,11, \mathrm{~A}, \mathrm{~B}, \mathrm{C}$, and D).

Our observations clearly confirm and extend the findings of Cunfer and Scolari (7), van den Mooter et al. (33), Azad and Schaad (1), Kersters et al. (13), Paul and Smith (23), Stead (31), Vauterin et al. $(37,34)$, and Bragard et al. $(3,4)$. The results reported here highlight the applicability and value of rep-PCR genomic fingerprints in classification and identification of $X$. translucens. Meaningful clusters are observed that are correlated to AFLP clusters and, in part, to the four pathogenicity profiles reported by Bragard et al. (3), and constitute a classification framework that can be used for identification of novel isolates.

Results obtained by conventional biochemical tests, Biolog, and FAME analysis did not conclusively assign 33 isolates from ornamental asparagus to the correct Xanthomonas sp. or subspecific group (22). This may have been due to the relatively low taxonomic resolution of the techniques and limitations of size and diversity of the libraries used. The rep-PCR genomic fingerprint database that we used to identify 33 novel isolates pathogenic to asparagus comprises over 300 isolates (29), including the 20 currently defined genospecies (34) and over 80 pathovars. The repPCR genomic fingerprint profiles of the novel isolates are highly similar to the profiles of rep-PCR cluster 1 (this article), which corresponds to AFLP group 1, for which the designation X. translucens pv. undulosa is proposed (3). This allows the conclusion that the 33 asparagus strains do belong to the redefined $X$. translucens pv. undulosa. The taxonomic placement of the asparagus strains in the genus Xanthomonas also was supported by their having an RsaI ARDRA profile characteristic for all Xanthomonas spp. The taxonomic position is further strengthened and detailed by the observation of an $X$. translucens-specific Msp I/ $A l u \mathrm{I}$ profile of the amplified SSU rDNA restriction profile that is in concordance with the comparison of $16 \mathrm{~S}$ rDNA sequences of all Xanthomonas spp. (12). As well as several phenotypic tests, the identification of the novel asparagus strains is based on five different DNA fingerprint profiling methods applied in this study (BOX-, ERIC-, and REP-PCR profiles and two ARDRA profiles). The independent fingerprinting methods have high confirmatory power, strengthening the individual observations as desired in a polyphasic taxonomic approach.

That the novel asparagus isolates belong to $X$. translucens $\mathrm{pv}$. undulosa is extraordinary because all hosts of $X$. translucens pathovars described so far consist of only cereals and grasses 
(families Gramineae and Poaceae), whereas asparagus belongs to a phylogenetically distant family (Liliaceae). This remarkable finding was supported by the observation that all isolates from ornamental asparagus were able to cause disease symptoms on wheat by mist inoculation. Out of 24 reference strains representing $9 X$. translucens pathovars, only $X$. translucens pv. arrhenatheri strain ATCC 33803 (=LMG 727) did not infect wheat. This pathogen, originally isolated from oat grass (A. elatius), has a small host range among forage grasses (strain 719 in reference 8). Surprisingly, several of the $X$. translucens reference strains also caused disease on asparagus tree fern. These pathogenicity tests show that our knowledge about the hosts of $X$. translucens is limited, particularly with regard to wild plant species or other plants of no or low economic importance. The novel strains were isolates from ornamental asparagus, an economically interesting crop. No correlation of the pathogenicity to asparagus of the $X$. translucens reference strains with original host, host range, or geographical origin was found.

The inoculation methods used for the plant pathogenicity tests represent different methods (wounding and nonwounding) by which the bacterial pathogens can infect the host. Pathogenicity results will vary between these inoculation procedures and should be considered to represent variation that would be seen naturally in the environment. The results of the plant pathogenicity tests on wheat using foil cap and syringe injection methods do not contribute significantly to the classification. This may be due to a low efficiency of provoking a pathogenicity response by these methods or to the smaller number of tests performed with these methods. The mist and foil inoculations were done in different laboratories, leading to some variation in incubation temperature, growth period, and media utilized, but none of these parameters varied enough to cause significant differences in the infection process.

Using four bacteriophage isolates pathogenic to X. translucens pv. translucens strains, only some of the 33 novel bacterial isolates reacted weakly to very weakly to one phage. The other three phages provoked no reactions. This result could be explained by a high specificity of the phages and may indicate that the novel asparagus isolates are epidemiologically unique compared with the other X. translucens strains tested. However, the phage testing was of little value for classification. It is not likely but it cannot be excluded that if more (reference) strains were tested, useful patterns would emerge.

The pathovar system or classification can be useful for the practitioner but is poorly defined due to variation in the hostpathogen interactions (8), and host specificity often is not clear. Pathogenicity tests are cumbersome, laborious, time consuming, and costly to carry out and have limited reproducibility. Moreover, isolates obtained from novel hosts or with an unclear taxonomic position that display a distinct pathogenicity are often difficult to classify at a pathovar level due to limitations in examining a comprehensive host range. Furthermore, host specificity coincides with different genomic backgrounds ([geno]species, PCR fingerprint types, or both), such as in xanthomonads associated with pepper and tomato lesions $(2,26,29,35)$ and rice $(6,26)$ that were differentiated with the advent of PCR-based genomic typing methods (15). Extensive examination of Xanthomonas spp. has shown that the phytopathogenic specialization of the bacteria is not correlated with the actual natural or phylogenetic relationships within the genus (36). The pathovar system, although useful when the strains have a small and specific host range, is of limited value when the host range is large and poorly defined. In these cases, genomic fingerprinting and gene sequence analyses become most relevant for taxonomy as well as its practical applications. For the classification of Xanthomonas spp. and, more specifically, the subspecific classification of $X$. translucens, repPCR and AFLP genomic fingerprints are most useful. Additional SSU rDNA sequence or restriction analysis types are highly practical to differentiate the $X$. translucens genomic lineage from the other Xanthomonas spp.

In conclusion, the unique $M s p \mathrm{I} / A l u \mathrm{I}$ SSU rDNA fingerprint profile specific to all $X$. translucens and the novel asparagus strains; the partial reciprocal plant test results; the BOX-, ERIC-, and REP-PCR; and combined genomic fingerprint analyses all support the placement of the novel strains in the $X$. translucens pv. undulosa group as proposed by Bragard et al. (3). Clearly, knowledge of the genomic background of bacteria in general, the genus Xanthomonas in particular and, more specifically, within $X$. translucens, as reflected in rep-PCR genomic fingerprints, is of great use in bacterial systematics and practical application.

\section{ACKNOWLEDGMENTS}

This work was supported by the DOE (DE FG 0290ER20021), the NSF Center for Microbial Ecology (DIR 8809640), and the Consortium for Plant Biotechnology Research (DE-FC05-02OR22072). We thank J. Wallace for excellent technical assistance.

\section{LITERATURE CITED}

1. Azad, H., and Schaad, N. W. 1988. Serological relationships among membrane proteins of strains of Xanthomonas campestris translucens. Phytopathology 78:272-277.

2. Bouzar, H., Jones, J. B., Stall, R. E., Louws, F. J., Schneider, M., Rademaker, J. L. W., de Bruijn, F. J., and Jackson, L. E. 1999. Multiphasic analysis of xanthomonads causing bacterial spot disease on tomato and pepper in the Caribbean and Central America: Evidence for common lineages within and between countries. Phytopathology 89:328-335.

3. Bragard, C., Singer, E., Alizadeh, A., Vauterin, L., Maraite, H., and Swings, J. 1997. Xanthomonas translucens from small grains: Diversity and phytopathological relevance. Phytopathology 87:1111-1117.

4. Bragard, C., Verdier, V., and Maraite, H. 1995. Genetic diversity among Xanthomonas campestris strains pathogenic to small grains. Appl. Environ. Microbiol. 661:1020-1026.

5. Bragard, C., and Verhoyen, M. 1993. Monoclonal antibodies specific for Xanthomonas campestris strains pathogenic to wheat and other small grain, in comparison with polyclonal antisera. J. Phytopathol. 139:217228.

6. Cruz, C. M. V., Ardales, E. Y., Skinner, D. Z., Talag, J., Nelson, R. J., Louws, F. J., Leung, H., Mew, T. W., and Leach, J. E. 1996. Measurement of haplotypic variation in Xanthomonas oryzae within a single field by rep-PCR and RFLP analyses. Phytopathology 86:1352-1359.

7. Cunfer, B. M., and Scolari, B. L. 1982. Xanthomonas campestris pv. translucens on triticale and other small grains. Phytopathology 72:683-686.

8. Egli, T., and Schmidt, D. 1982. Pathogenic variation among the casual agents of bacterial wilt of forage grasses. Phytopathol. Z. 104:138-150.

9. Forster, R. L., and Strausbaugh, C. A. 1994. Use of bacteriophage for identification of Xanthomonas campestris pv. translucens. In: Proc. 8th Int. Conf. Plant Pathogenic Bacteria. M. LeMattre, S. Freigoun, K., Rudolph, and J. G. Swings, eds. INRA, Versailles, France.

10. Forster, R. L., Strausbaugh, C. A., and Schaad, N.W. 1995. Modified XTS agar for isolation of Xanthomonas campestris pv. translucens from wheat and barley seed. (Abstr.) Phytopathology 85:1182.

11. Häne, B. G., Jager, K., and Drexler, H. G. 1993. The Pearson productmoment correlation-coefficient is better suited for identification of DNA fingerprint profiles than band matching algorithms. Electrophoresis 14:967-972.

12. Hauben, L., Vauterin, L., Swings, J., and Moore, E. R. B. 1997. Comparison of $16 \mathrm{~S}$ ribosomal DNA sequences of all Xanthomonas species. Int. J. Syst. Bacteriol. 47:328-335.

13. Kersters, K., Pot, B., Hoste, B., Gillis, M., and De Ley, J. 1989. Protein electrophoresis and DNA:DNA hybridizations of xanthomonads from grasses and cereals. EPPO Bull. 19:51-55.

14. King, E. O., Ward, M. K., and Raney, D. E. 1954. Two simple media for the demonstration of pyocyanin and fluorescein. J. Lab. Clin. Med. 44:301-307.

15. Louws, F. J., Rademaker, J. L. W., and de Bruijn, F. J. 1999. The three Ds of PCR-based genomic analysis of phytobacteria: Diversity, detection, and disease diagnosis. Annu. Rev. Phytopathol. 37:85-125.

16. MacFaddin, J. F. 1981. Biochemical Tests for Identification of Medical Bacteria. 2nd ed. Williams and Wilkins, Baltimore/London.

17. Maes, M., Garbeva, P., and Kamoen, O. 1996. Recognition and detection in seed of the Xanthomonas pathogens that cause cereal leaf streak using rDNA spacer sequences and polymerase chain reaction. Phytopathology 86:63-69. 
18. Milus, E. A., and Mirlohi, A. F. 1993. A test tube assay for estimating populations of Xanthomonas campestris pv. translucens on individual wheat leaves. Phytopathology 83:134-139.

19. Moore, E. R. B., Kruger, A. S., Hauben, L., Seal, S. E., Debaere, R., Dewachter, R., Timmis, K. N., and Swings, J. 1997. 16S rRNA gene sequence analyses and inter- and intrageneric relationships of Xanthomonas species and Stenotrophomonas maltophilia. FEMS Microbiol. Lett. 151:145-153.

20. Moyer, C. L., Tiedje, J. M., Dobbs, F. C., and Karl, D. M. 1996. A computer simulated restriction fragment length polymorphism analysis of bacterial smallsub-unit rRNA genes: Efficacy of selected tetrameric restriction enzymes for studies of microbial diversity in nature. Appl. Environ. Microbiol. 62:2501-2507.

21. Norman, D., and Alvarez, A. 1989. A rapid method for the presumptive identification of Xanthomonas campestris pv. dieffenbachiae and other xanthomonads. Plant Dis. 73:654-658.

22. Norman, D. J., Yuen, J. M. F., and Hodge, N. C. 1997. New disease on ornamental asparagus caused by Xanthomonas campestris in Florida. Plant Dis. 81:847-850.

23. Paul, V. H., and Smith, I. M. 1989. Bacterial pathogens on gramineae: Systematic view and assessment of quarantine status for the EPPO region. EPPO Bull. 19:33-42.

24. Pierce, L., and Schroth, M. N. 1994. Detection of Pseudomonas colonies that accumulate poly- $\beta$-hydroxybutyrate on Nile Blue Medium. Plant Dis. 78:683-685.

25. Rademaker, J. L. W., and de Bruijn, F. J. 1997. Characterization and classification of microbes by rep-PCR genomic fingerprinting and computer assisted pattern analysis. Pages 151-171 in: DNA Markers, Protocols, Applications and Overviews. G. Ceatano-Anollés and P. M. Gresshoff, eds. J. Wiley \& Sons, New York.

26. Rademaker, J. L. W., Hoste, B., Louws, F. J., Kersters K., Swings, J., Vauterin, L., Vauterin, P., and de Bruijn, F. J. 2000. Comparison of AFLP and rep-PCR genomic fingerprinting with DNA-DNA homology studies: Xanthomonas as a model system. Int J. Syst. Bacteriol. 50:665-677.

27. Rademaker, J. L. W., Louws, F. J., and de Bruijn, F. J. 1998. Characterization of the diversity of ecological important microbes by rep-PCR genomic fingerprinting. Pages 1-26 in: Molecular Microbial Ecology Manual, Supplement 3. A. D. L. Akkermans J. D. van Elsas, and F. J. de Bruijn, eds. Kluwer Academic Publishers, Dordrecht, The Netherlands.

28. Rademaker, J. L. W., Louws, F. J., Rossbach, U., Vinuesa, P., and de Bruijn, F. J. 1999. Computer-assisted pattern analysis of molecular fingerprints and database construction. Pages 1-33 in: Molecular Microbial
Ecology Manual, Supplement 4. A. D. L. Akkermans, J. D. van Elsas, and F. J. de Bruijn, eds. Kluwer Academic Publishers, Dordrecht, The Netherlands.

29. Rademaker, J. L. W., Louws, F. J., Schultz, M. H., Rossbach, U., Vauterin, L., Swings, J., and de Bruijn, F. J. 2005. A comprehensive species to strain taxonomic framework for Xanthomonas. Phytopathology 95:10981111 .

30. Sambrook, J., Fritsch, E. F., and Maniatis, T. 1989. Molecular Cloning: A Laboratory Manual. Cold Spring Harbor Laboratory, Cold Spring Harbor, NY.

31. Stead, D. E. 1989. Grouping of Xanthomonas campestris pathovars of cereals and grasses by fatty acid profiling. Bull. OEPP/EPPO Bull. 19:5768.

32. Suslow, T. V., Schroth, M. N., and Isaka, M. 1982. Application of a rapid method for gram-differentiation of plant pathogenic and saprophytic bacteria without staining. Phytopathology 72:917-918.

33. van den Mooter, M., Steenackers, M., Maertens, C., Gossele, F., De Vos, P., Swings, J., Kersters, K., and de Ley, J. 1987. Differentiation between Xanthomonas campestris pv. graminis ISPP L 1980, pv. phleipratensis ISPP List 1980 emend., pv. poae Egli an Schmidt 1982, and pv. arrhenatheris Egli and Schmidt 1982, by numerical analysis of phenotypic features and protein gel electrophoresis. J. Phytopathol. 118:135-156.

34. Vauterin, L., Hoste, B., Kersters, K., and Swings, J. 1995. Reclassification of Xanthomonas. Int. J. Syst. Bacteriol. 45:472-489.

35. Vauterin, L., Rademaker, J. L. W., and Swings, J. 2000. Synopsis on the taxonomy of Xanthomonas. Phytopathology 90:677-682.

36. Vauterin, L., and Swings, J. 1997. Are classification and phytopathological diversity compatible in Xanthomonas? J. Ind. Microbiol. Biotechnol. 19:77-82.

37. Vauterin, L., and Vauterin, P. 1992. Computer aided objective comparison of electrophoretic pattern for grouping and identification of microorganisms. Eur. Microbiol. 1:37-41.

38. Vauterin, L., Yang, P., Hoste, B., Pot, B., Swings, J., and Kersters, K. 1992. Taxonomy of xanthomonads from cereals and grasses based on SDS-PAG proteins, fatty acid analysis and DNA hybridization. J. Gen. Microbiol. 138:1467-1477.

39. Versalovic, J., Schneider, M., de Bruijn, F. J., and Lupski, J. R. 1994. Genomic fingerprinting of bacteria using repetitive sequence-based polymerase chain reaction. Methods Mol. Cell. Biol. 5:25-40.

40. Wilson, E. E., Zeitoun F. M., and Fredrickson, D. L. 1967. Bacterial phloem canker, a new disease of Persian walnut trees. Phytopathology 57:618-621. 